\title{
Differences in physical characteristics, perinatal histories, and social backgrounds between children with growth hormone deficiency and constitutional short stature
}

\author{
G V VIMPANI, A F VIMPANI, S J POCOCK, AND J W FARQUHAR \\ Department of Child Life and Health, University of Edinburgh
}

SUMMARY Four hundred and forty-nine children with he:ghts below $-2 \cdot 5$ SD were ident fied by screening for height a total population of 48221 in three Scottish cities. Children participating in the study could be classified into 5 groups: severe growth hormone deficiency $(n=13)$, partial growth hormone deficiency $(n=25)$, low birthweight short stature $(n=34)$, constitutional short stature $(\mathrm{n}=178)$, and short stature associated with some underlying disease $(\mathrm{n}=106)$. Children with growth hormone deficiency tended to be shorter, were more likely to be obese, were growing more slowly, more often were the products of an abnormal pregnancy, and were less socially disadvantaged than constitutionally short children. Their parents were also more likely to have sought medical advice about the short stature. These findings have important implications for improved casefinding of children suffering from growth-hormone deficiency, who in this study accounted for about $10 \%$ of all short but otherwise normal children who came from non-disadvantaged communities.

Previous reports ${ }^{2}$ of the differences in physical characteristics between children with growthhormone $(\mathrm{GH})$ deficiency and constitutional short stature have been based on very short children referred to hospital clinics and may have been unrepresentative of such children generally. Any differences may therefore have been affected by bias. The present report is based on a cohort of very short children identified by screening a defined population of schoolchildren for short stature. The primary objective of the study was to determine the cause of short stature in every child but this paper reports on differences in physical characteristics, perinatal histories, and social backgrounds that were noted between children with GH deficiency and those with constitutional short stature.

\section{Materials and method}

For the purposes of this study, children were defined as having short stature if their height standard deviation (SD) score, based on the standards of Tanner et al. ${ }^{3}$ was $₹-2 \cdot 5$. Height SD scores were calculated as follows:

$$
\begin{aligned}
\text { SD score } & =\mathbf{X}-\overline{\mathbf{X}} / \mathrm{SD} \\
\mathbf{X} & =\text { child's height }(\mathrm{mm}) \\
\overline{\mathrm{X}}, \mathbf{S D}= & \text { mean and standard deviation } \\
& \text { appropriate for age and sex. }
\end{aligned}
$$

The heights of all 2nd- and 3rd-year primary pupils attending education authority schools and some attending independent schools in Edinburgh, Glasgow, and Aberdeen were screened during 5 months in 1975-76. In Aberdeen 4th-year pupils too were screened. (An unknown (but small) amount of within-city migration took place during the screening, particularly in Glasgow, where measurements took 3 months to complete, which resulted in some children being measured twice and others not at all.)

With shoes removed, each child was measured while standing in the classroom, by one of the investigators (GVV) using a Harpenden portable anthropometer. Any child with severe physical handicap was measured lying down. The smallest children in each age group were then measured again by the same investigator, this time using the technique recommended by Tanner et al. ${ }^{1}$ with a wallmounted stadiometer transported in a purpose-built 
mobile clinic. The accuracy of the stadiometer was checked at each school, and the reliability of the measurements was confirmed by finding that when 28 children from one school were measured on successive days, $95 \%$ of repeat heights were within $4.5 \mathrm{~mm}$.

The numbers of children in each class and the numbers absent were obtained from the teacher who also was able to give personal details of the children measured with a stadiometer. Schools were visited again so as to measure any absentee thought by his teacher to be small.

Attempts were then made to get into contact with the parents of all children with short stature. The initial contact was by post, and any non-responder was followed up personally and told about the study and the possible benefits for the child. With parental consent, information about appreciable medical conditions was sought from general practitioners and paediatricians who had treated the child.

Later, generally at the child's school, details of any previous major illness or medical investigation on the child were obtained by interviewing one parent (often the mother) or both of them. This interview, carried out by one of three speciallytrained interviewers, was based on a previously tested precoded interview schedule. It also sought to elicit information about the child's perinatal history and social background. Parents were asked if they had been concerned about their child's short stature and whether they had sought medical advice about it.

From information obtained at interview a 'social disadvantage score' was derived for each child based on indicators of social disadvantage previously described. ${ }^{19}$ One point was given for the presence of each of the following, resulting in a minimum score of $\mathbf{0}$ and a maximum score of 3: (a) only one parent, or five or more children in family; (b) low income family - that is family had at some stage been in receipt of Supplementary Benefit, or the child had been eligible for free school meals; (c) poor quality housing-that is more than 1.5 persons per room, or no exclusive use of hot water.

At the same time the child underwent a careful physical and anthropometric examination. The height of one parent, or if possible both, was measured to the nearest centimetre using the anthropometer. All anthropometry was done by one observer (GVV). Triceps and subscapular skinfold measurements were made with Harpenden skinfold calipers. ${ }^{4}$ Occipitofrontal head circumference and mid-upper arm circumference were measured with a flexible steel tape measure, and mid-upper arm muscle circumference was derived from the formula given by Jelliffe. ${ }^{5}$
Upper arm muscle $=$ upper arm circumference (mm)

Circumference $(\mathrm{mm})-\pi \times$ triceps skinfold

thickness (mm)

Most children were seen on several occasions during the course of the study enabling an annual height velocity to be calculated, but to lessen any seasonal effect on growth rate only those in whom the height velocity was derived from measurement intervals in excess of $\mathbf{0 . 8 7 5}$ years were included in the analysis.

All raw anthropometric data were converted to an SD or centile score by making use of established standards. For height, the cross-sectional standards of Tanner ${ }^{6}$ were used. Skinfold thickness was expressed as a centile ranking derived from the revised British standards of Tanner and Whitehouse. ${ }^{4}$ Upper arm muscle circumference was calculated as a percentage of the mean given by Jelliffe ${ }^{5}$ as no British standards are thought to exist. Because the distribution of height velocity is not gaussian over the whole age range of the cohort, each height velocity was accorded a centile ranking ${ }^{6}$ rather than an SD score.

In the absence of previous investigations or any evident organic basis for short stature, informed consent to carry out a battery of diagnostic tests was sought from the parents. These tests included postexercise serum growth hormone, ${ }^{7}$ thyroxine, thyroidstimulating hormone, urea, 25-hydroxycholecalciferol, calcium, phosphorous, white cell count, urine culture (girls), and chromosome analysis (girls). Unless the parents refused, any child with a postexercise GH level of less than $10 \mathrm{mU} / 1$ or any child with a level less than $18 \mathrm{mU} / 1$ in whom the height velocity over 12 months was less than the 25 th centile for chronological age was admitted to hospital for an insulin hypoglycaemia test. All GH investigations were done in the same laboratory to satisfy high quality control criteria. ${ }^{7}$ An $x$-ray film of the left wrist and hand was also taken for subsequent bone age estimation ${ }^{8}$ by a trained reader (AFV). Results pertaining to many of these investigations will be reported elsewhere.

If the mean and SD scores of variables in the diagnostic groups could be derived, between-group comparisons were made using Student's $t$ test, unless small skewed samples were being compared in which case the Mann-Whitney $U$ test was used.

If a child had been assigned to a centile ranking for a particular variable, a between-group comparison was made using a test for trend in proportions. ${ }^{18}$ The test statistic (c) is normally distributed and its significance can be read from tables of the normal distribution.

If binary variables were examined, between-group 
comparisons were made using a $\chi^{2}$ test for two independent samples (corrected for continuity if appropriate).

\section{Results}

Four hundred and forty-nine children with short stature aged between 6.03 and 9.36 years were identified in a total screened population of 48221 (Table 1). Of these, 108 (24\%) children had an organic basis (other than GH deficiency) for the short stature (Table 2). Only 26 of these children were investigated in the study, the underlying organic basis for the short stature being newly diagnosed in only two (a girl with hypothyroidism and one who was a Turner's mosaic). Another was a boy with acute lymphoblastic leukaemia who was only partially investigated by an exercise test (GH 4.8 $\mathrm{mU} / \mathrm{l})$ before he suffered an unrelated relapse and died. In the other 82 children the nature of the disease was thought sufficient to exclude the need for further investigations. The parents of a further

Table 1 Short stature-numbers and prevalence rates per 1000 by city, gender, and type of school

\begin{tabular}{|c|c|c|c|c|}
\hline & Edinburgh & Glasgow & Aberdeen & Total \\
\hline \multicolumn{5}{|l|}{ Primary schools } \\
\hline $\begin{array}{l}\text { Number } \\
\% \\
\text { Girls }\end{array}$ & $\stackrel{26}{4 \cdot 47}$ & $\begin{array}{l}159 \\
12 \cdot 03\end{array}$ & $\begin{array}{l}13 \\
2 \cdot 91\end{array}$ & $\begin{array}{r}198 \\
8 \cdot 42\end{array}$ \\
\hline $\begin{array}{l}\text { Number } \\
\% \\
\text { Total }\end{array}$ & $\begin{array}{l}30 \\
5 \cdot 30\end{array}$ & $\begin{array}{r}119 \\
9.48\end{array}$ & $\stackrel{24}{5 \cdot 66}$ & $\begin{array}{r}173 \\
7 \cdot 71\end{array}$ \\
\hline $\begin{array}{l}\text { Number } \\
\% \\
\text { Special schools }\end{array}$ & $\begin{array}{l}56 \\
4 \cdot 88\end{array}$ & $\begin{array}{l}278 \\
10 \cdot 8\end{array}$ & $\begin{array}{l}37 \\
4 \cdot 25\end{array}$ & $\begin{array}{r}371 \\
8.07\end{array}$ \\
\hline Boys & & & & \\
\hline $\begin{array}{l}\text { Number } \\
\% \\
\text { Girls }\end{array}$ & $\begin{array}{r}8 \\
129\end{array}$ & $\begin{array}{l}26 \\
158 \cdot 5\end{array}$ & $\stackrel{9}{154 \cdot 2}$ & $\begin{array}{c}43 \\
149 \cdot 3\end{array}$ \\
\hline $\begin{array}{l}\text { Number } \\
\% \\
\text { Total }\end{array}$ & $\begin{array}{c}5 \\
116 \cdot 3\end{array}$ & $\begin{array}{c}16 \\
116 \cdot 8\end{array}$ & $\begin{array}{c}12 \\
230 \cdot 8\end{array}$ & $\begin{array}{c}33 \\
142 \cdot 2\end{array}$ \\
\hline $\begin{array}{l}\text { Number } \\
\% \\
\text { Independent schools } \\
\text { Boys }\end{array}$ & $\begin{array}{c}13 \\
123 \cdot 8\end{array}$ & $\begin{array}{l}42 \\
139 \cdot 5\end{array}$ & $\begin{array}{c}21 \\
184 \cdot 2\end{array}$ & $\begin{array}{c}76 \\
146 \cdot 2\end{array}$ \\
\hline $\begin{array}{l}\text { Number } \\
\% \\
\text { Girls }\end{array}$ & - & - & - & - \\
\hline $\begin{array}{l}\text { Number } \\
\% \\
\text { Total }\end{array}$ & - & $\begin{array}{l}1 \\
3 \cdot 01\end{array}$ & $\begin{array}{l}1 \\
8 \cdot 77\end{array}$ & $\begin{array}{l}2 \\
2 \cdot 29\end{array}$ \\
\hline $\begin{array}{l}\text { Number } \\
\% \\
\text { All schools }\end{array}$ & - & $\begin{array}{l}1 \\
1 \cdot 95\end{array}$ & $\begin{array}{l}1 \\
5 \cdot 08\end{array}$ & $\begin{array}{l}2 \\
1 \cdot 14\end{array}$ \\
\hline Boys & & & & \\
\hline $\begin{array}{l}\text { Number } \\
\% \\
\text { Girls }\end{array}$ & $\begin{array}{l}34 \\
5 \cdot 23\end{array}$ & $\begin{array}{l}185 \\
13 \cdot 6\end{array}$ & $\begin{array}{l}22 \\
4 \cdot 77\end{array}$ & $\begin{array}{l}241 \\
9 \cdot 77\end{array}$ \\
\hline $\begin{array}{l}\text { Number } \\
\% \\
\text { Grand total }\end{array}$ & $\begin{array}{l}35 \\
5 \cdot 72\end{array}$ & $\begin{array}{l}136 \\
10 \cdot 4\end{array}$ & $\begin{array}{l}37 \\
8 \cdot 39\end{array}$ & $\begin{array}{l}208 \\
8 \cdot 83\end{array}$ \\
\hline $\begin{array}{l}\text { Number } \\
\%\end{array}$ & $\begin{array}{l}69 \\
5.47\end{array}$ & $\begin{array}{l}321 \\
12 \cdot 08\end{array}$ & $\begin{array}{l}59 \\
6 \cdot 54\end{array}$ & $\begin{array}{r}449 \\
9 \cdot 31\end{array}$ \\
\hline
\end{tabular}

$77(17 \%)$ children with short stature refused to participate in the study, but the remaining 264 children had either been adequately investigated previously $(\mathrm{n}=10)$ or underwent investigation in this study $(n=254)$.

Table 2 Short stature associated with organic disease excluding $G H$ deficiency in 108 children (mean height SD scores, and range in brackets)

\begin{tabular}{|c|c|}
\hline $\begin{array}{l}\text { Neurological abnormalities }(\mathrm{n}=39) \\
\text { Spina bifida }(-3 \cdot 7 ;-2 \cdot 5 \text { to }-5 \cdot 6) \\
\text { Cerebral palsy }(-3 \cdot 2 ;-2 \cdot 5 \text { to }-5 \cdot 1) \\
\text { Microcephaly }(-3 \cdot 0,-3 \cdot 2,-4 \cdot 0) \\
\text { Arrested hydrocephalus }(-2 \cdot 6,-2 \cdot 7) \\
\text { Epilepsy and severe mental retardation }(-3 \cdot 4,-4 \cdot 6) \\
\text { Hemiplegia }(-2 \cdot 5) \\
\text { Neurofibromatosis }(-3 \cdot 3) \\
\text { Meningitis and nerve deafness } \ddagger(-2 \cdot 7) \\
\text { Non-specific severe mental retardation }(-3 \cdot 0,-3 \cdot 2)\end{array}$ & $\begin{array}{r}14 \\
13 \\
3 \\
2\left(2^{*}\right) \\
2 \\
1\left(1^{*}\right) \\
1\left(1^{*}\right) \\
1\left(1^{*}\right) \\
2\end{array}$ \\
\hline $\begin{array}{l}\text { Skeletal abnormalities }(\mathrm{n}=17) \\
\text { Scoliosis }(-2 \cdot 8 ;-2 \cdot 5 \text { to }-3 \cdot 1) \\
\text { Achondroplasia }(-4 \cdot 8,-4 \cdot 3,-5 \cdot 5) \\
\text { Achondroplasia and spina bifida occulta }(-5 \cdot 2) \\
\text { Hypochondroplasia }(-4 \cdot 5) \\
\text { Sacral tumour }(-5 \cdot 3,-4 \cdot 8) \\
\text { Perthes’s disease }(-3 \cdot 2,-2 \cdot 6) \\
\text { Klippel-Feil abnormality }(-3 \cdot 1) \\
\text { Metaphyseal dysostosis }(-3 \cdot 3) \\
\text { Rickets } ¥(-3 \cdot 7)\end{array}$ & $\begin{array}{l}5\left(1^{*}\right) \\
3 \\
1 \\
1 \\
2 \\
2\left(2^{*}\right) \\
1 \\
1\left(1^{*}\right) \\
1\left(1^{*}\right)\end{array}$ \\
\hline $\begin{array}{l}\text { Chromosomal abnormalities }(\mathrm{n}=17) \\
\text { Down's syndrome }(-3 \cdot 2 ;-2 \cdot 5 \text { to }-4 \cdot 2) \\
\text { 'Trisomy } 18 \text { ' }(-4 \cdot 0) \\
\text { Turner's mosaic }(45, \mathrm{XO} / 46, \mathrm{Xi}(\mathrm{Xq}))\end{array}$ & $\begin{array}{r}15 \\
1 \\
1\left(1^{*}\right)\end{array}$ \\
\hline $\begin{array}{l}\text { Respiratory disorders } \\
\text { Asthma }(-2 \cdot 7 ;-2 \cdot 5 \text { to }-3 \cdot 4) \\
\text { Chronic bronchitis }(-2 \cdot 7,-3 \cdot 3) \\
\text { Cystic fibrosis }(-2 \cdot 5)\end{array}$ & $\begin{array}{ll}5 & \left(2^{*}\right) \\
2 & \left(2^{*}\right) \\
1 & \end{array}$ \\
\hline $\begin{array}{l}\text { Cardiovascular disorders }(\mathrm{n}=6) \\
\text { Pulmonary stenosis }(-3 \cdot 1,-2 \cdot 6,-2 \cdot 8) \\
\text { Ventricular septal defect }(-3 \cdot 7) \\
\text { Patent ductus arteriosus }(-3 \cdot 2) \\
\text { Intersex and chronic heart disease }(-4 \cdot 3)\end{array}$ & $\begin{array}{ll}3 & \left(2^{*}\right) \\
1 & \left(1^{*}\right) \\
1 & \\
1 & \end{array}$ \\
\hline $\begin{array}{l}\text { Gastrointestinal abnormalities }(\mathrm{n}=6) \\
\text { Coeliac disease }(-3 \cdot 1,-3 \cdot 2,-2 \cdot 6) \\
\text { Hirchsprung's disease }(-3 \cdot 7) \\
\text { Imperforate anus and mental retardation }(-3 \cdot 2) \\
\text { Tracheo-oesophageal fistula }(-6 \cdot 3)\end{array}$ & $\begin{array}{l}3\left(1^{*}\right) \\
1 \\
1 \\
1\end{array}$ \\
\hline $\begin{array}{l}\text { Metabolic and storage disorders }(n=4) \\
\text { Glycogen storage disease }(-2 \cdot 9) \\
\text { Hurler's syndrome }(-5 \cdot 6) \\
\text { Morquio's syndrome }(-4 \cdot 8) \\
\text { Non-specific storage disorder }(-3 \cdot 2)\end{array}$ & $\begin{array}{ll}1 & \left(1^{*}\right) \\
1 & \\
1 & \end{array}$ \\
\hline $\begin{array}{l}\text { Haematological disorders }(\mathrm{n}=2) \\
\text { Acute lymphoblastic leukemia }(-2 \cdot 9) \\
\text { Chronic idiopathic thrombocytopenic purpura }(-2 \cdot 2) \dagger\end{array}$ & $\begin{array}{l}1\left(1^{*}\right) \\
1\end{array}$ \\
\hline $\begin{array}{l}\text { Renal disorders }(\mathrm{n}=2) \\
\text { Renal hypoplasia }(-5 \cdot 1) \\
\text { Severe recurrent urinary tract infections }(-3 \cdot 5)\end{array}$ & $\begin{array}{l}1\left(1^{*}\right) \\
1\left(1^{*}\right)\end{array}$ \\
\hline $\begin{array}{l}\text { Endocrine abnormalities }(\mathrm{n}=2) \\
\text { Hypothyroidism }(-2 \cdot 6) \\
\text { Gonadal hypoplasia }(-3 \cdot 2)\end{array}$ & $\begin{array}{l}1\left(1^{*}\right) \\
1\end{array}$ \\
\hline $\begin{array}{l}\text { Miscellaneous }(\mathrm{n}=5) \\
\text { Russell-Silver syndrome }(-3 \cdot 5,-4 \cdot 0) \\
\text { Ataxia telangiectasia }(-2 \cdot 4) \\
\text { Progeria }(-4 \cdot 7) \\
\text { Smaller of discordant twins }(-3 \cdot 7)\end{array}$ & $\begin{array}{l}2\left(1^{*}\right) \\
1 \\
1 \\
1(1 *)\end{array}$ \\
\hline
\end{tabular}

Neurological abnormalities $(\mathrm{n}=39)$

Spina bifida $(-3 \cdot 7 ;-2 \cdot 5$ to $-5 \cdot 6)$

Arrested hydrocephalus $(-2 \cdot 6,-2 \cdot 7)$

(lepsy and severe mental retardation $(-3.4,-4.6)$

Hemiplegia $(-2 \cdot 5)$

Non-specific severe mental retardation $(-3 \cdot 0,-3 \cdot 2)$

Hypochondroplasia $(-4 \cdot 5)$

cral tumour $(-5 \cdot 3$,

Metaphyseal dysostosis $(-3 \cdot 3)$

'Trisomy 18' $(-4.0)$

Turner's mosaic (45, XO/46, Xi(Xq))

Cardiovascular disorders $(n=6)$

Pulmonary stenosis $(-3 \cdot 1,-2 \cdot 6,-2 \cdot 8)$

$(-4 \cdot 3)$

alities $(n=6)$

Coeliac disease $(-3 \cdot 1,-3 \cdot 2,-2 \cdot 6)$

Tracheo-oesophageal fistula $(-6 \cdot 3)$

Metabolic and storage disorders $(n=4)$

Non-specific storage disorder $(-3 \cdot 2)$

Haematological disorders $(n=2)$

Severe recurrent urinary tract infections $(-3 \cdot 5)$

(1*)

Hypothyroidism $(-2 \cdot 6)$

Ataxia telangiectasia $(-2 \cdot 4)$

Progeria $(-4 \cdot 7)$

Smaller of discordant twins $(-3 \cdot 7)$

when all subjects were $<-2 \cdot 50$. 
On the basis of perinatal, auxological, and biochemical criteria it was possible to classify all but 14 of these 264 children into 4 groups. Each of the 14 children who could not be so classified had a post-exercise GH level of $<18 \mathrm{mU} / 1$ and a height velocity of less than the 25 th centile, but because of emigration $(n=1)$ or refusal by the parents, did not undergo an insulin hypoglycaemia test. Although GH deficiency could not be positively excluded it is possible that other influences-such as low birthweight, or genetic factors-might have been responsible for the short stature.

\section{Classification}

Group 1, severe GH deficiency ( $n=13,8$ boys).

Short stature, maximum GH level after exercise and during insulin hypoglycaemia test $₹ 9.9 \mathrm{mU} / 1$.

Group 2, partial $G H$ deficiency $(n=25,17$ boys).

Short stature, maximum GH level after exercise and during insulin hypoglycaemia test $₹ 15.9 \mathrm{mU} / \mathrm{l}$.

All were from Glasgow.

Group 3, low birthweight short stature $(n=34,24$ boys).

Short stature, GH deficiency excluded, birthweight $<5$ th centile for gestational age and maternal height, ${ }^{9}$ no other organic disease.
Group 4, constitutional short stature $(n=178,90$ boys).

Short stature, GH deficiency excluded, birthweight $>5$ th centile for gestational age and maternal height, no other organic disease.

All but 11 met Tanner and Thomson's criteria for a diagnosis of short/delay. ${ }^{9}$ Most $(75 \%)$ came from Glasgow.

Tables 3 and 4 show the auxological, perinatal, and social background characteristics of these 4 groups. It is clear that the children with GH deficiency were shorter, were growing more slowly, had thicker skinfolds, and had less muscle bulk than those with constitutional short stature. Although boys with GH deficiency were noticeably smaller than their constitutionally short counterparts, girls with GH deficiency were no shorter than those with constitutional short stature. The children with GH deficiency were more likely to have been born post-term, to mothers aged under 20, who had had a complicated pregnancy, and to have had a normal birthweight than those with constitutional short stature. They also tended to be members of numerically smaller families suffering less from overcrowding and social disadvantage than the constitutionally short group. Additionally their parents were more likely to have sought medical advice

Table 3 Auxological differences in children with severe or partial GH deficiency, low birthweight short stature, and constitutional short stature

\begin{tabular}{|c|c|c|c|c|c|}
\hline \multirow[t]{2}{*}{ Characteristic } & \multicolumn{2}{|l|}{ Growth hormone deficiency } & \multirow{2}{*}{$\begin{array}{l}\text { Low birthweight } \\
\text { short stature } \\
\text { Group } 3(n=34)\end{array}$} & \multirow{2}{*}{$\begin{array}{l}\text { Constitutional } \\
\text { short stature } \\
\text { Group } 4(n=178)\end{array}$} & \multirow{2}{*}{$\begin{array}{l}\text { Significance } \\
\text { difference } \\
\text { between } \\
\text { groups } 1 \text { and } 4\end{array}$} \\
\hline & $\begin{array}{l}\text { Severe } \\
\text { Group I }(n=13)\end{array}$ & $\begin{array}{l}\text { Partial } \\
\text { Group } 2(n=25)\end{array}$ & & & \\
\hline $\begin{array}{l}\text { Mean height* } \\
\text { (+ range) }\end{array}$ & $-3 \cdot 39(-2 \cdot 50 \rightarrow-4 \cdot 84)$ & $-2 \cdot 95(-2 \cdot 55 \rightarrow-4 \cdot 11)$ & $-2 \cdot 70(-2 \cdot 07 \rightarrow-3 \cdot 63)$ & $-2 \cdot 77(-2 \cdot 10 \rightarrow-3 \cdot 88)$ & $\begin{array}{l}t=5.76 \\
\mathrm{P}<0.01\end{array}$ \\
\hline Mean height* & $\mathrm{n}=8$ & $\mathrm{n}=17$ & $\mathrm{n}=24$ & $\mathrm{n}=24$ & \\
\hline $\begin{array}{c}\text { (+ range) (boys) } \\
\text { Mean height SD score* }\end{array}$ & $\begin{array}{l}-3 \cdot 83(-2 \cdot 72 \rightarrow-4 \cdot 84) \\
n=5\end{array}$ & $\begin{array}{l}-2 \cdot 94(-2 \cdot 55 \rightarrow-3 \cdot 50) \\
n=8\end{array}$ & $\begin{array}{l}-2 \cdot 68(-2 \cdot 07 \rightarrow-3 \cdot 88) \\
n=10\end{array}$ & $\begin{array}{l}-2 \cdot 73(-2 \cdot 21 \rightarrow-3 \cdot 88) \\
n=88\end{array}$ & $\mathbf{P}<0.01$ \\
\hline (+ range) (girls) & $\begin{array}{l}n=5 \\
-2 \cdot 69(-2 \cdot 50 \rightarrow-2 \cdot 91)\end{array}$ & $\begin{array}{l}n=8 \\
-2 \cdot 96(-2 \cdot 57 \rightarrow-4 \cdot 11)\end{array}$ & $\begin{array}{l}n=10 \\
-2 \cdot 77(-2.41 \rightarrow-3 \cdot 63)\end{array}$ & $\begin{array}{l}n=88 \\
-2 \cdot 81(-2 \cdot 10 \rightarrow-3 \cdot 64)\end{array}$ & NS \\
\hline $\begin{array}{l}\text { Percentage with height } \\
\text { velocity }<25 \text { th } \\
\text { centile }\end{array}$ & $\begin{array}{l}\mathrm{n}=9 \dagger \\
89\end{array}$ & $\begin{array}{l}\mathrm{n}=25 \\
80\end{array}$ & $\begin{array}{l}\mathrm{n}=27 \\
59\end{array}$ & $\begin{array}{l}\mathrm{n}=138 \\
61\end{array}$ & $\begin{array}{l}\text { Test for trend } \\
c=3.25 \\
P=0.001\end{array}$ \\
\hline Bone age SD score & $\begin{array}{l}\mathrm{n}=10 \\
-1 \cdot 87( \pm 0.95)\end{array}$ & $\begin{array}{l}n=25 \\
-2 \cdot 29( \pm 1 \cdot 06)\end{array}$ & $\begin{array}{l}\mathrm{n}=34 \\
-1 \cdot 83( \pm 1 \cdot 11)\end{array}$ & $\begin{array}{l}\mathrm{n}=173 \\
-1 \cdot 75( \pm 1 \cdot 00)\end{array}$ & NS \\
\hline $\begin{array}{l}\text { Percentage with } \\
\text { triceps skinfold }\end{array}$ & & & & & $\begin{array}{l}\text { Test for trend } \\
\mathrm{c}=2.55\end{array}$ \\
\hline $\begin{array}{l}\text { thickness less than } \\
25 \text { th centile }\end{array}$ & 15 & 40 & 56 & 44 & $P=0.01$ \\
\hline $\begin{array}{l}\text { Percentage with upper } \\
\text { arm muscle } \\
\text { circumference less } \\
\text { than } 90 \% \text { of }\end{array}$ & & & & & $\begin{array}{l}\text { Test for trend } \\
\quad c=2.44 \\
P=0.015\end{array}$ \\
\hline standard & 69 & 40 & 29 & 30 & \\
\hline $\begin{array}{l}\text { Head circumference } \\
\text { SD score (mean } \pm \text { SD } \\
\text { Maternal height SD } \\
\text { score }( \pm \text { SD) }\end{array}$ & $\begin{array}{l}n=12 \\
n-0 \cdot 6( \pm 0 \cdot 96) \\
n=13 \\
-1 \cdot 44( \pm 1 \cdot 54)\end{array}$ & $\begin{array}{l}\mathrm{n}=25 \\
-1 \cdot 21( \pm 1 \cdot 24) \\
\mathrm{n}=25 \\
-1 \cdot 75( \pm 0 \cdot 76)\end{array}$ & $\begin{array}{l}n=33 \\
-1 \cdot 29( \pm 0 \cdot 86) \\
n=34 \\
1 \cdot 27( \pm 1 \cdot 06)\end{array}$ & $\begin{array}{l}n=178 \\
-1 \cdot 17( \pm 1 \cdot 10) \\
n=169 \\
-1 \cdot 94( \pm 0 \cdot 76)\end{array}$ & $\begin{array}{l}t=1.75 \\
\mathrm{P}=0.1 \\
t=2.08 \\
\mathrm{P}<0.05\end{array}$ \\
\hline
\end{tabular}

* This height SD score was calculated at time of examination and investigation, generally some months after the initial height screening, when all subjects were $<-2 \cdot 50$.

$\dagger$ Three children previously diagnosed and on treatment (12-month height velocity not available in other case). 
Table 4 Perinatal and social background characteristics of children with severe or partial GH deficiency, low birthweight stature, and constitutional short stature

\begin{tabular}{|c|c|c|c|c|c|}
\hline \multirow[t]{2}{*}{ Characteristic } & \multicolumn{2}{|c|}{ Growth hormone deficiency } & \multirow{2}{*}{$\begin{array}{l}\text { Low birthweight } \\
\text { short stature, } \\
\text { group } 3\end{array}$} & \multirow{2}{*}{$\begin{array}{l}\text { Constitutional } \\
\text { short stature, } \\
\text { group } 4\end{array}$} & \multirow{2}{*}{$\begin{array}{l}\text { Significance of } \\
\text { differences betweer } \\
\text { groups } 1 \text { and } 4\end{array}$} \\
\hline & $\begin{array}{l}\text { Severe } \\
\text { group } 1\end{array}$ & $\begin{array}{l}\text { Partial } \\
\text { group } 2\end{array}$ & & & \\
\hline $\begin{array}{l}\text { Pregnancy order, percentage in which subject } \\
\text { resulted from first pregnancy }\end{array}$ & $38(5 / 13)$ & $8(2 / 15)$ & $12(4 / 34)$ & $17(33 / 171)$ & $\begin{array}{l}\text { Test for trend } \\
c=1.62 \\
P=0.1\end{array}$ \\
\hline $\begin{array}{l}\text { Percentage of mothers aged }<20 \\
\text { at birth of first child }\end{array}$ & $67(8 / 12)$ & $48(/ 25)$ & $65(22 / 34)$ & $55(94 / 172)$ & NS \\
\hline $\begin{array}{l}\text { Percentage of mothers aged }<20 \\
\text { at birth of subject }\end{array}$ & $67(8 / 12)$ & $12(3 / 25)$ & $12(4 / 34)$ & $23(39 / 172)$ & $\begin{array}{l}\chi^{2}=9.22 \\
\mathbf{P}<0.01\end{array}$ \\
\hline $\begin{array}{l}\text { Percentage with abnormal pregnancy } \\
\text { history* }\end{array}$ & $69(9 / 13)$ & $32(8 / 25)$ & $41(14 / 34)$ & $30(51 / 168)$ & $\begin{array}{l}x^{2}=6.57 \\
P=0.01\end{array}$ \\
\hline Percentage with abnormal delivery & $42(5 / 12)$ & $20(5 / 25)$ & $12(4 / 34)$ & $16(28 / 170)$ & NS \\
\hline $\begin{array}{l}\text { Percentage born post-term ( } 42 \text { or more } \\
\text { weeks' gestation) }\end{array}$ & $31(4 / 13)$ & $28(7 / 25)$ & $12(4 / 34)$ & $10(17 / 177)$ & $\begin{array}{l}\chi^{2}=-3.67 \\
P=0.06\end{array}$ \\
\hline $\begin{array}{l}\text { Percentage of mothers who smoked in } \\
\text { pregnancy }\end{array}$ & $42(5 / 12)$ & $68(17 / 25)$ & $79(27 / 34)$ & $57(98 / 171)$ & $\begin{array}{l}x^{2}=4.93 \dagger \\
P<0.05\end{array}$ \\
\hline Mean birthweight (g) \pm SD & $\begin{array}{l}n=13 \\
3238 \pm 613\end{array}$ & $\begin{array}{l}n=24 \\
2865 \pm 482\end{array}$ & $\begin{array}{l}n=34 \\
2334 \pm 339\end{array}$ & $\begin{array}{l}n=164 \\
2970 \pm 480\end{array}$ & $\begin{array}{l}t=1.90 \\
P<0.1\end{array}$ \\
\hline Mean family size \pm SD & $\begin{array}{l}n=13 \\
3 \cdot 23 \pm 1 \cdot 48\end{array}$ & $\begin{array}{l}n=25 \\
4.92+1.85\end{array}$ & $\begin{array}{l}n=34 \\
4 \cdot 64 \pm 2 \cdot 14\end{array}$ & $\begin{array}{l}n=172 \\
4.68 \pm 1.95\end{array}$ & $\begin{array}{l}t=2.62 \\
\mathrm{P}<0.01\end{array}$ \\
\hline $\begin{array}{l}\text { Percentage from families with } 5 \text { or more } \\
\text { children }\end{array}$ & $0(0 / 13)$ & $36(9 / 25)$ & $24(8 / 34)$ & $24(49 / 172)$ & $\begin{array}{l}\text { Test for trend } \\
c=2.63 \\
P=0.008\end{array}$ \\
\hline $\begin{array}{l}\text { Percentage from households living at } \\
\text { density }>1.5 \text { persons per room }\end{array}$ & $15(2 / 13)$ & $68(17 / 25)$ & $44(15 / 34)$ & $54(93 / 172)$ & $\begin{array}{l}\text { Test for trend } \\
\mathrm{C}=3 \cdot 32 \\
\mathrm{P}=0.001\end{array}$ \\
\hline $\begin{array}{l}\text { Percentage from households in which father } \\
\text { unemployed }\end{array}$ & $23(3 / 13)$ & $32(8 / 25)$ & $44(15 / 34)$ & $29(50 / 172)$ & NS \\
\hline $\begin{array}{l}\text { Percentage with social disadvantage score } \\
\text { of } 3\end{array}$ & $8(1 / 13)$ & $33(8 / 24)$ & $26(9 / 34)$ & $31(53 / 170)$ & $\begin{array}{l}\text { Test for trend } \\
\mathbf{c}=1.99 \\
\mathbf{P}=0.05\end{array}$ \\
\hline $\begin{array}{l}\text { Percentage of mothers concerned } \\
\text { by subject's short stature }\end{array}$ & $69(9 / 13)$ & $56(14 / 25)$ & $35(12 / 34)$ & $42(71 / 171)$ & $\begin{array}{l}\chi^{2}=2 \cdot 73 \\
\mathbf{P}=0 \cdot 1\end{array}$ \\
\hline $\begin{array}{l}\text { Percentage who were thought to be smaller } \\
\text { than siblings }\end{array}$ & $82(9 / 11)$ & $76(19 / 25)$ & $65(22 / 34)$ & $69(113 / 164)$ & NS \\
\hline $\begin{array}{l}\text { Percentage whose parents had sought medical } \\
\text { advice }\end{array}$ & $69(9 / 13)$ & $28(7 / 25)$ & $18(6 / 34)$ & $18(31 / 170)$ & $\begin{array}{l}x^{2}=15 \cdot 52 \\
\mathbf{P}<0.001\end{array}$ \\
\hline
\end{tabular}

* Specific questions about swelling/PET, vaginal blood loss, severe vomiting, or other complications. $\dagger$ Group 3 compared with group 4 .

about the child's short stature than those of the constitutionally short children.

One-third of the constitutionally short children were severely socially disadvantaged by our criteria. Since this group comprised the bulk of the study population it was not surprising to find that this group was closely linked with the social and economic environment. For instance, short stature was twice as common in Glasgow as in Edinburgh or Aberdeen, and was 7 times more common in children attending education authority schools than in those attending independent schools. Moreover within Glasgow, where there was a 20 -fold variation in the rate of short stature between different postal districts (Figure) a significant correlation $(\mathrm{Rs}=$ $0.73, \mathrm{P}<0.01$ ) was found between the prevalence of short stature and the frequency of previously described census indicators of urban deprivation. ${ }^{10-12}$ In Edinburgh (Figure) where organic causes of short stature were more common, the correlation was less pronounced and failed to reach statistical significance $(R s=0.41, P<0.1)$.
Constitutional short stature was often associated with mental retardation as has been found by others. ${ }^{13}$ For instance, in Glasgow, schools for the

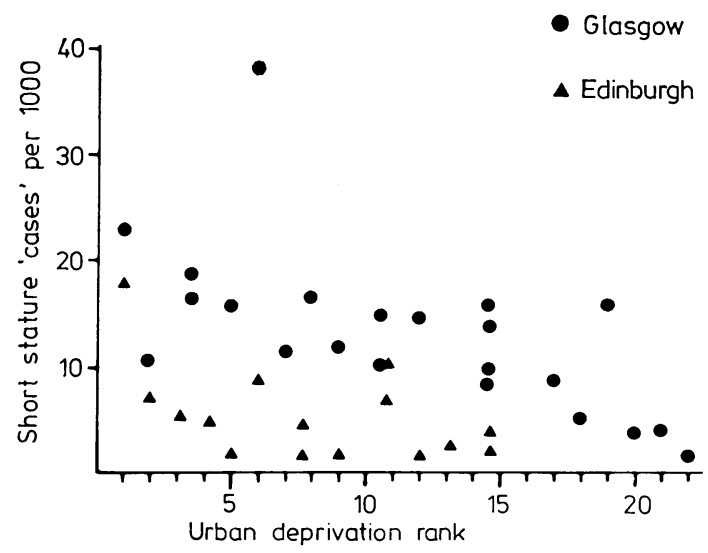

Figure Relationship between short stature and deprivation in Glasgow and Edinburgh. 
educationally subnormal were attended by only $0.58 \%$ of the population but accounted for $4.4 \%$ of all short children. Furthermore, in Glasgow a further 14 children with short stature were transferred to schools for the educationally subnormal because of their inability to cope with normal learning.

In this study children with low birthweight short stature were only slightly different from those with constitutional short stature. Indeed the only statistically significant difference between the groups in any of these parameters was the fact that a higher proportion of mothers of children of low birthweight had smoked cigarettes during the relevant pregnancy.

The range of height and height velocity in the 77 non-participating children was similar for those with constitutional short stature. Only $14 \%$ had heights $\gtrless-3$ SD scores; only one girl (with a normal height velocity) was $<-4$ SD score.

\section{Discussion}

The results of this study show some of the auxological, perinatal, and social background factors which tend to distinguish children with GH deficiency from those with constitutional short stature. A chubby, normally intelligent, short child of normal birthweight, more often the product of a pregnancy marked by complications, who comes from a comfortable home is more likely to be GH deficient than a lean, sometimes dull, underweight child from poor circumstances. The perinatal differences noted here are consistent with those reported earlier by Rona and Tanner ${ }^{14}$ who noted an increased risk of GH deficiency in first-born children, those born after a breech delivery, or in those born after vaginal bleeding during pregnancy.

While we noted that there were some appreciable differences between groups of children with $\mathrm{GH}$ deficiency and constitutional short stature with respect to auxological and other characteristics, prediction of the correct diagnosis in each case on the basis of these is not always possible; this confirms the observation of Joss ${ }^{2}$ who noted that parameters such as height and height velocity did not invariably distinguish between children with $\mathbf{G H}$ deficiency and those with constitutional short stature.

Unlike those with severe GH deficiency, children with partial deficiency are virtually indistinguishable from those with constitutional short stature in terms of perinatal and social background characteristics. This finding raises serious questions about the significance and aetiology of such children below normal GH responses. Is it valid to consider them as a separate diagnostic group? Probably only a randomised, controlled therapeutic trial can answer that question. It is worth noting that 6 had other conditions which might have contributed to the short stature-such as coeliac disease, which has previously been reported to be associated with depressed $\mathrm{GH}$ responses, ${ }^{15}$ and one each had asthma, probable fetal alcohol syndrome, and Russell-Silver syndrome. Could it also be that social deprivation ${ }^{16}$ is an additional trigger to those described by Rona and Tanner ${ }^{14}$ and capable of producing depressed GH levels in those with a genetic susceptibility to GH deficiency?

Although previous estimates of the prevalence of GH deficiency have ranged from 1 in 30000 to 1 in $10000^{13} 1417$ we found the prevalence to be about 1 in 4000 . Our results clearly indicate that professional inactivity rather than a lack of parental concern was responsible for many of the missed diagnoses in this series. For instance the parents of children with GH deficiency were more likely to seek medical advice than the parents of constitutionally short children, but fewer than half (4 out of 9) had appropriate diagnostic investigations initiated. If all 9 had been investigated case-finding by practitioners independent of this study would have been doubled. These findings bear out the adage 'If parents suspect something is wrong, it probably is'. GH deficiency is one of the most common organic causes of short stature. In a non-disadvantaged community (for example Aberdeen or Edinburgh) it may account for $5 \%$ of all cases of short stature $(₹-2.5 \mathrm{SD}$ score), or $10 \%$ of cases if those with a known organic basis for their short stature are excluded.

We thank the teachers and parents who made the study possible; Professor J O Forfar, Professor J Hutchinson, and Professor A Campbell for cooperation; Dr W Hamilton and Dr M Fyfe for the use of their hospital facilities; Dr Euan Cameron and his staff, Regional Hormone Laboratory, Edinburgh, for growth hormone analyses; Professor $\mathbf{J} \mathbf{M}$ Tanner for invaluable advice and encouragement.

The Medical Research Council provided financial support.

\section{References}

1 Tanner J M, Whitehouse R H, Hughes P C R, Vince F P. Effect of human growth hormone treatment for 1 to 7 years on growth of 100 children with growth hormone deficiency, low birthweight, inherited smallness, Turner's syndrome, and other complaints. Arch Dis Child 1971; 46: 745-82.

2 Joss E E. Growth hormone deficiency in childhood: evaluation of diagnostic procedures. Monographs in 
Paediatrics No 5. New York: Karger, 1975: 618-905; M 75.

3 Tanner J M, Whitehouse R H, Takaishi M. Standards from birth to maturity for height, weight, height velocity, and weight velocity: British children, 1965. Part I. Arch Dis Child 1966; 41: 454-71.

4 Tanner J M, Whitehouse R H. Revised standards for triceps and subscapular skinfolds in British children. Arch Dis Child 1975; 50: 142-5.

5 Jelliffe D B. The assessment of the nutritional status of the community (with special reference to field surveys in developing regions of the world). WHO Monogr Ser 1966; 53: 237.

- Tanner J M. Physical growth and development. In: Forfar J O, Arneil G, eds. Textbook of paediatrics. Edinburgh: Churchill Livingstone, 1973: 224-91.

7 Vimpani G V, Vimpani A F, Lidgard G P, Cameron E H D, Farquhar J W. Prevalence of severe growth hormone deficiency. Br Med J 1977; ii: 427-30.

8 Tanner J M, Whitehouse R H, Marshall W A, et al. Assessment of skeletal maturity and prediction of adult height (TW2 method). London: Academic Press, 1975.

9 Tanner J M, Thomson A M. Standards for birthweight at gestation periods from 32 to 42 weeks, allowing for maternal height and weight. Arch Dis Child 1970; 45: 566-9.

10 Levein C P A, McKenzie A. Summary report of an investigation to identify areas of multiple deprivation in Glasgow city. CPRU Working Paper No 7. Glasgow: Scottish Development Department, 1973: 1-7.

11 Holterman S. Areas of urban deprivation in Great Britain: an analysis of 1971 census data. In: Thompson E J,
Lewis C, eds. Social trends. No 6. London: CSO/HMSO, 1975: 33-47.

12 Vimpani G V. A community study of short stature with special reference to the prevalence of severe growth hormone deficiency. PhD thesis, University of Edinburgh 1977.

13 Lacey K A, Parkin J M. Causes of short stature. A community study of children in Newcastle upon Tyne. Lancet 1974; i: 42-5.

14 Rona R J, Tanner J M. Aetiology of idopathic growth hormone deficiency in England and Wales. Arch Dis Child 1977; 52: 197-208.

15 Vanderschueren-Lodeweyckx M, Wolter R, Molla A, Eggermont E, Eeckels R. Plasma growth hormone in coeliac disease. Helv Paediatr Acta 1973; 28: 349-57.

16 Powell G F, Brasel J A, Blizzard R M. Emotional deprivation and growth retardation simulating idiopathic hypopituitarism. I. Clinical evaluation of the syndrome. N Engl J Med 1967; 276: 1271-8.

17 Lacey K A, Parkin J M. The normal short child. Community study of children in Newcastle upon Tyne. Arch Dis Child 1974; 49: 417-24.

18 Cox D R. Analysis of binary data. London: Methuen, 1970.

19 Wedge P, Prosser H. Born to fail. London: Arrow Books, 1973.

Correspondence to Professor J W Farquhar, Department of Child Life and Health, University of Edinburgh, 17 Hatton Place, Edinburgh EH9 1UW.

Received 16 December 1980

Received 16 December 1980 